\title{
Fine-Grained Radio Resource Management to Control Interference in Dense Wi-Fi Networks
}

\author{
M. Seyedebrahimi, F. Bouhafs, A. Raschellà, M. Mackay, Q. Shi \\ Department of Computer Science \\ Liverpool John Moores University \\ Liverpool, U.K. \\ \{M.Seyedebrahimi, F.Bouhafs, A.Raschella, M.I.Mackay, Q.Shi\}@ljmu.ac.uk
}

\begin{abstract}
In spite of the enormous popularity of Wi-Fienabled devices, the utilisation of Wi-Fi radio resources (e.g. RF spectrum and transmission power levels) at Access Points (APs) is degraded in current decentralised Radio Resource Management (RRM) schemes. Most state of the art centralised control solutions apply configurations in which the network-wide impacts of the involved parameters and their mutual relationships are ignored. In this paper, we propose an algorithm for jointly adjusting the transmission power levels and optimising the $R F$ channel assignment of APs by taking into account the flows' required qualities while minimising their interference impact throughout the network. The proposed solution is tailored for an operatoragnostic and Software Defined Wireless Networking (SDWN)based centralised RRM in dense Wi-Fi networks. Our extensive simulation results validate the performance improvements of the proposed algorithm compared to the main state of the art alternative by showing more than $\mathbf{2 5 \%}$ higher spectrum efficiency, satisfying the users' demands and further mitigating the networkwide interference through flow-based and quality-oriented power level adjustment.
\end{abstract}

Keywords-Wireless LAN; Radio Resource Management; RF Channel Assignment; Transmission Power Control; SDWN

\section{INTRODUCTION}

The last few years have witnessed a massive increase in the popularity of portable devices, such as smartphones and tablets, thanks to their functionality, user-friendly interface, and affordable price. This situation has created a growing demand on the wireless spectrum in general, but more specifically on $\mathrm{Wi}$ Fi. Due to its utilisation of non-licensed frequency bands, Wi-Fi is now facing significant spectrum efficiency issues especially in dense areas, such as apartment blocks and shopping malls, where neighbouring Wi-Fi Access Points (APs) interfere with each other while competing for the spectrum. This interference between Wi-Fi networks can negatively affect users' experience, resulting in lower throughput and poor quality connections.

Radio interference in wireless networks has long been a major research challenge that has been studied extensively in the literature [1]-[8]. Certain contributions have adopted a per-cell approach that addressed the interference problem through dynamic channel assignment. In [1] for example, the authors proposed an approach where an AP can select a suitable configuration based on the channel monitoring and traffic status obtained locally and decided centrally for each AP. In [2], the authors proposed a heuristic algorithm that assigns a channel to an AP by analysing the effect of partially overlapping channels on the Wi-Fi network throughput. In [3], the authors formulated the optimisation of channel assignment as a graph colouring problem. However, these solutions do not consider other performance-related measurements at the AP such as Signal to Interference plus Noise Ratio (SINR) and interference levels. Although these network-side measurements have been considered in the contributions presented in [4] and [5], the impact of these measurements on the performance of the solutions proposed is limited as they are taken locally and not across all of the interference environment.

Other contributions have adopted a per-client approach by focusing on adjusting the transmission power of the communication link between the wireless station (STA) and the AP. In Wi-Fi networks, STAs typically transmit at an output power level which is adopted and decided locally given their required quality. This potentially generates an unwanted level of interference on the corresponding channel. In [6], the authors considered a combination of power level control and rate adjustment for meeting the link quality requirements. Rate selection is determined by an estimation of the channel conditions including packet loss, delivery ratio, throughput, or SINR estimation. Moreover, in [7], the authors investigate the probability distribution of users' optimal power levels and exploit this for defining and broadcasting the desired power levels to the user. This approach, however, requires the users' involvement in the process. In [8] the authors consider the minimisation of the interference level when different APs and WLANs are working in the same area, while not affecting the performance perceived by the client. However, this approach needs GPS synchronisation, which is a significant weakness for indoor deployments.

Software-Defined Networking (SDN) [9] has emerged as an efficient and flexible network management approach for large networks. By decoupling the control plane from the data plane, SDN can centralise the network management operations in a single entity, often referred to as a Controller. This centralised management approach allows us to remotely programme large networks through the OpenFlow protocol [9]. These features have recently attracted the attention of the wireless communication research community, who have launched a range of initiatives to develop open source Software Defined Wireless Networking (SDWN) frameworks to manage Wi-Fi networks such as EmPOWER [10], Odin [11], and Wi-5 [12][13] which all aim to extend Openflow to support Wi-Fi network management operations and radio configuration primitives.

In this paper, we propose a fine-grained Radio Resource Management (RRM) algorithm that focuses on Wi-Fi APs, and 
exploits the programmability and centralised management capabilities offered by SDWN to dynamically configure these devices. The proposed algorithm is based on a per-flow approach that takes into account the application flow Quality of Service (QoS) requirements, as well as the effect of the radio configuration in the Wi-Fi APs, on the rest of the network. To the best of our knowledge there have been no previous contributions that combine dynamic channel assignment and transmit power control, and takes into account QoS to mitigate the network-wide interference in dense Wi-Fi networks.

The rest of this paper is structured as follows. Our SDWNbased Wi-Fi management framework and design assumptions are presented in Section II. In Section III, we introduce a quantity which represents the impact of each source of interference in our proposed channel assignment optimization problem and also provides a network-wide benchmark for interference evaluation. In Section IV we highlight certain considerations toward the fine-grained RRM algorithm and explain the details of the proposed heuristic. In Section V we assess our proposed algorithm and illustrate the obtained results. Finally our conclusions are presented in Section VI.

\section{SDWN-BASED WI-FI MANAGEMENT FRAMEWORK}

The algorithm presented in this paper represents part of the work carried out in the $\mathrm{H} 2020$ funded $\mathrm{Wi}-5$ project, which aims to address spectrum congestion in Wi-Fi networks. The project proposes an architecture [12] that acknowledges the heterogeneity of Wi-Fi network operators and uses SDWN to provide a coordination platform that could be used to implement cooperative spectrum management algorithms [14], [15], as illustrated in Fig. 1. The choice of SDWN to build a cooperative Wi-Fi network management platform is justified by the centralised nature of this concept which offers the operators, and any entity that manages a Wi-Fi AP, including households, an interface through which a cooperative spectrum utilisation policy could be agreed. Moreover, SDWN offers flexibility and cross-layer management, as the central controller is able to obtain monitoring information about the status of the network and execute the cooperative algorithms to react accordingly while respecting the requirements of the wireless users, such as the solution presented in [14]. It is worth mentioning that Wi-5 is currently developing a SDWN framework that extends the capabilities of OpenFlow to support the monitoring of $\mathrm{Wi}-\mathrm{Fi}$

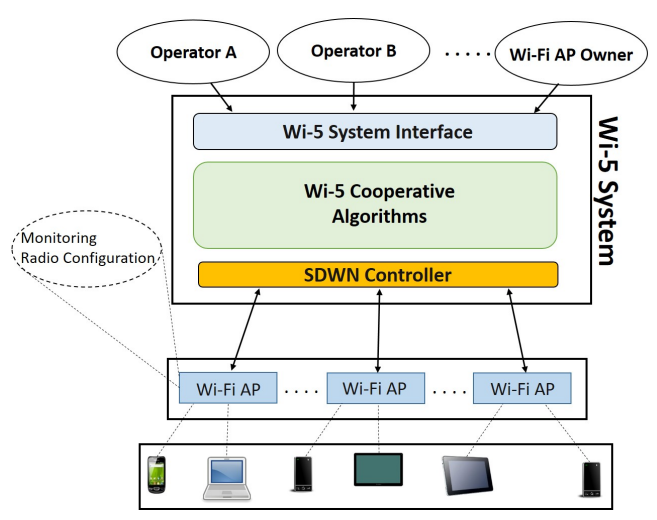

Fig. 1: Wi-Fi network management architecture in Wi-5 project networks, the QoS requirements of wireless applications, and the configuration of Wi-APs [13].

In addition to the distinct advantages of an SDWN-based RRM approach for Wi-Fi networks, the corresponding algorithm, as presented in this paper, provides the capabilities which are required in a multi-operator, dynamic and partially controllable network. These capabilities are: 1) a practical way to include/exclude any network element of interest from the realtime process, 2) representing the included APs and their mutual impacts through a network-wide quality metric, 3) capturing the mutual relationship between radio resource parameters and the APs distribution throughout the network, and 4) taking into account the correlation between the employed network-wide quality metric and the per-user's quality demand.

\section{NETWORK-WIDE INTERFERENCE ANALYSIS}

In this section we first revisit our channel assignment optimisation problem, which has preliminarily been introduced in [15]. We then extend the analysis of its interference-related quantity, interference impact, as a basis for providing a networkwide quality indicator from the perspective of interference. This will later be merged with a flow-based and quality-oriented power adjustment mechanism to establish our proposed RRM algorithm. For the rest of the paper, the following network arrangements are assumed:

- We consider $N$ Wi-Fi APs, based on the IEEE 802.11 standard, that operate on $F$ RF channels including $F_{\text {non }}$ of them not-overlapping each other.

- We assume $N>F_{n o n}$, i.e. there is channel overlapping and therefore an interference problem in the network. An example is $F=11$ and $F_{\text {non }}=3$ in the IEEE $802.112 .4 \mathrm{GHz}$ band where $\mathrm{N} \geq 4$ is the starting point of channel overlapping and the densification problem.

- We assume that APs are the only elements transacting with the information required at the central controller. APs are the sensing points and measurement agents for the central controller throughout the network and the ultimate configuration is applied to APs via the downlink.

\section{A. Channel Assignment Optimisation}

In [15] the channel assignment optimisation problem is defined as follows:

$$
A^{*}=\min _{A} \sum_{i \leq N} \sum_{\mathrm{f} \leq \mathrm{F}} G \times A^{T} . I
$$

where $\mathrm{G}^{\mathrm{N} \times \mathrm{N}}$ is defined as the network topology matrix $G \in$ $\{0,1\}^{N \times N}$, where:

$$
g_{i j}=\left\{\begin{array}{r}
1, \quad \begin{array}{r}
\text { average power strength of } A P_{i} \text { around } A P_{j} \\
\text { exceeds a given threshold }
\end{array} \\
0, \quad \text { otherwise }
\end{array}\right.
$$

and $\mathrm{A}^{\mathrm{F} \times \mathrm{N}}$ as the channel assignment matrix $A \in\{0,1\}^{F \times N}$, where:

$$
a_{i j}=\left\{\begin{array}{lc}
1, & \text { if channel } i \text { is assigned to } A P_{j} \\
0, & \text { otherwise }
\end{array}\right.
$$


$I \in \mathbb{R}^{N \times F}$ is defined as the matrix of the interference impacts for $N$ APs and $F$ available channels, where each $I_{i, f}$ element as an interference impact, is the summation of the signals corresponding to channel $f$ when it is assigned to $A P_{i}$ and detected at other APs' locations. The interference impact for $A P_{i}$ and its corresponding channel $f$ can be expressed as follows:

$$
\begin{aligned}
& I_{i, f}=\sum_{k \leq N, k \neq i} P_{i, k}(f)=\sum_{k \leq N, k \neq i} P_{i}^{t} \gamma_{i, k}(f) \theta_{i, k}(f)= \\
& \quad P_{i}^{t} \sum_{k \leq N, k \neq i} \gamma_{i, k}(f) \theta_{i, k}(f)
\end{aligned}
$$

Where $1 \leq f \leq F, 1 \leq i, k \leq N, P_{i, k}$ is the average power strength of the RF channel assigned to $A P_{i}$ and sensed at close proximity of $A P_{k} . P_{i}^{t}$ is the transmission power level at $A P_{i}, \gamma_{\mathrm{i}, \mathrm{k}}$ is the channel gain between $A P_{i}$ and $A P_{k}$, and $\theta_{i, k}$ are coefficients varying from 0 to 1 representing the overlap between the channels assigned to $A P_{i}$ and $A P_{k}$. This coefficient will be zero for non-overlapping channels. An example of such overlap is provided in [3]. Both $\gamma_{i, k}$ and $\theta_{i, k}$ are, obviously, dependent on $f$. All values are estimated and updated in real-time and are dependent on the actual characteristics of the employed RF channels as well as the arrangement of the network.

The matrix $I$ reflects the interference impacts of APs' transmission powers in the objective function of (1) considering the overlap and orthogonality of the RF channels. The resulting optimised channel assignment, $A^{*}$, is supposed to minimise the summation of these impacts throughout the network. More details about the employed binary Integer Linear Programing (ILP) approach to solve (1) and the exact ILP coefficients can be found in [15].

\section{B. The Network-wide Interference Impact Quantity}

To examine the optimality of the solution provided in (1), we need to show that the experienced interference in the network with the channel assignment based on $A^{*}$, is lower than the interference by any other channel assignment combination. Let $I_{a c c}$ be the accumulation of the interferences which can be experienced at all the APs' locations. We aim to use this later in the proposed algorithm to represent the network-wide quality. This, by definition, is actually the summation of the signals detectable at an AP's location and originated from all other APs:

$$
I_{a c c}=\sum_{i=1}^{N} \sum_{k \leq N, k \neq i}^{N} P_{k}^{t} \gamma_{k, i} \theta_{k, i}
$$

$P_{i}^{t}, \gamma_{\mathrm{i}, \mathrm{k}}$ and $\theta_{i, k}$ are the same as in (4) and we drop symbol $f$ to avoid notation clutter. Given the resemblance between $i$ and $k$ 's range of values in (5) and comparing with the indexes in (4), their recast yields:

$$
\begin{array}{r}
I_{a c c}=\sum_{i=1}^{N} \sum_{k \leq N, k \neq i}^{N} P_{k}^{t} \gamma_{k, i} \theta_{k, i}= \\
\sum_{i=1}^{N} \sum_{k \leq N, k \neq i}^{N} P_{i}^{t} \gamma_{i, k} \theta_{i, k}=\sum_{i=1}^{N} I_{i, f_{i}}
\end{array}
$$

Where $f_{i}$ denotes the instance of $f$ which corresponds to $A P_{i}$. Since the linear summation with positive integer coefficients of all $I_{i, f}$ in (1) has already been shown to be optimal for $A^{*}$, for any linear summation with unit coefficients we have:

$$
\left.\sum_{i=1}^{N} I_{i, f_{i}}\right|_{A=A^{*}} \leq\left.\sum_{i=1}^{N} I_{i, f_{i}}\right|_{A=A^{\prime}}
$$

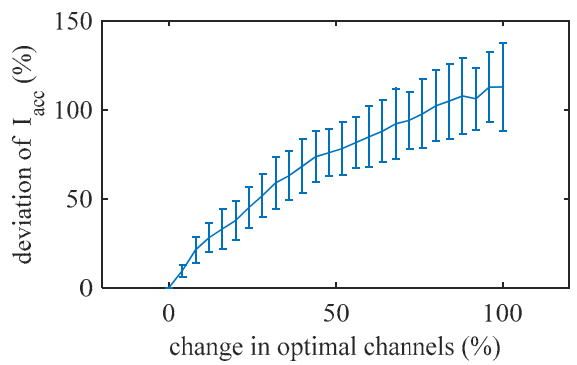

Fig. 2: deviation of the accumulated interference when the optimised channels are changing

Where $A$ ' refers to any channel assignment with at least one allocated channel different from $A^{*}$ and all $I_{i, f}$ are positive and greater than the threshold in (2). Applying (7) to (6) means that:

$$
\left.I_{a c c}\right|_{A=A^{*}} \leq\left. I_{a c c}\right|_{A=A^{\prime}}
$$

This shows that the status of the interference throughout the network and measured from APs point of view will be in its optimal situation immediately after applying the channel assignment $A^{*}$. We take this as a reference point for our algorithm in Section IV. Fig. 2 depicts an example of deviation from the optimal $I_{a c c}$ when the assigned channels are different from the optimal assignment. The illustrated result shows a positive and increasing trend of deviation from the optimal interference value for more changes compared to the APs optimal channel assignment. This is the validation of the optimality of $A^{*}$. A threshold, denoted as $\delta$, for acceptable deviation from the optimal interference value will be used, beyond which the channel assignment process will be triggered or the intended change will be denied.

\section{Fine-Grain Radio Resource Management ALGORITHM}

The network-wide interference quantity defined in (6) results in a direct relationship between the accumulated interference, $I_{a c c}$, discussed in Section III-B and the transmission power levels of the APs. Fig. 3 shows an example of the correlation between the AP transmission power levels and $I_{a c c}$. Color-coded values are depicted at the locations of the APs with each colour representing the assigned RF channel to that AP. The value represents the correlation between the transmission power level of that AP and $I_{a c c}$. The correlation values are all positive and close to 1 , which highlight a strong direct relationship.

However, the impacts of the APs vary based on their locations and/or their assigned channels. For instance, channel 1 assigned to the central location denoted as 0.98/1-red has a correlation value of 0.98 . This is higher than the correlation shown for its neighbouring AP (denoted as 0.72/4 green) because they are on different channels. The same central AP has a higher correlation compared to its co-channel AP at the upper left-side of the network (denoted as $0.79 / 1$ red) because of their different locations. These variations highlight the importance of capturing the mutual relationship between radio resource parameters and the APs distribution throughout the network in our proposed approach. 


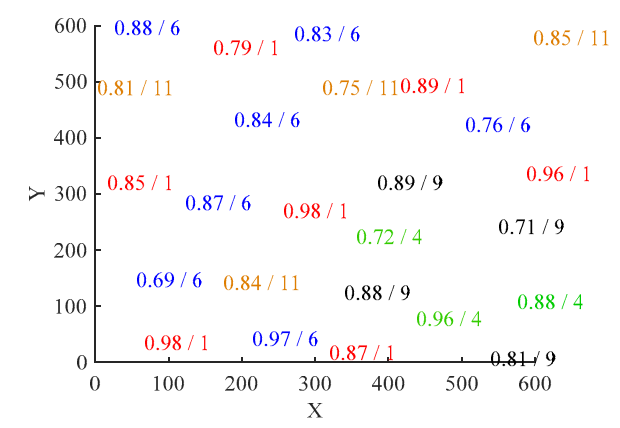

Fig. 3: correlation between the power level and accumulated interference under impacts of locations and assigned channels

The transmission power levels of APs are also positively affecting the transmission rates of their corresponding downlink flows, which in turn affects the provided QoS. This opposing impact of the transmission power level over the flow-based served quality, compared to their impact over the network-wide interference, needs to be addressed in a joint quality-oriented and flow-based power adjustment scheme alongside networkwide interference control. Assuming that the QoS requirements of the flows are known, the controller needs to adjust the transmission power of the APs such that the QoS requirements are satisfied and the level of interference in the network is maintained close to its optimal value.

Fig. 4 depicts the block diagram of our RRM strategy implemented on top of the SDWN controller, which can maintain the trade-off between the interference status in a dense Wi-Fi network and the satisfactory power levels for all of the flows joining the APs. The main tasks designed for the proposed algorithm are as follows:

J0: optimising the AP's assigned channel given the latest status of the network and setting a reference value for the optimal network-wide interference status. This is based on the optimisation model in (1). J1: estimating the flow's achievable rates in all available APs for a desired range of transmission power levels. This is conducted considering the provided service for other flows already associated with the APs, the estimation of the flow's channel status and the employed OFDM modulations and code rates. J2: taking the flow's required quality into account and assessing the impact on the networkwide interference. J3: assessing the impact of the settings for a new flow over currently active flows in the network, and selecting the most suitable AP for the service.

Algorithm 1 depicts the running sequence of these jobs in the implemented algorithm for the SDWN controller. First, the controller acquires all the measurements from the APs (line 1 in Algorithm 1) and then executes step J0 making use of (1) (line 2 in Algorithm 1). For each new flow connecting to the network, the controller assesses all the available APs that can be associated to the flow based on their Received Signal Strengths (RSS) (lines 3 and 4 in Algorithm 1). It then executes steps J1 and J2 for each AP (lines 5-11 in Algorithm 1). The controller then executes step J3 to select an AP for association (lines 12-13 in Algorithm 1) and finally runs J0 if flagged for the selected AP (lines 14 in Algorithm 1). Given $N$, the number of APs and $M$, the number of discrete applicable power levels, the main 'for

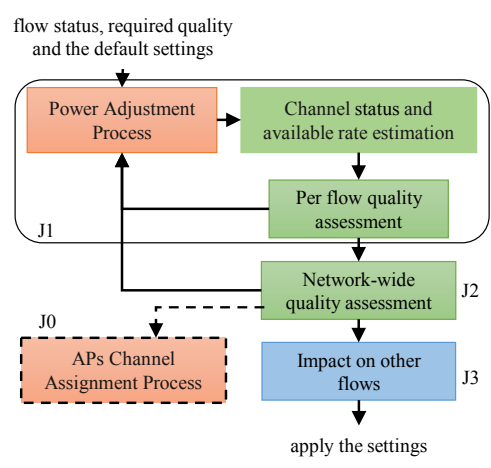

Fig. 4: joint power adjustment and channel assignment

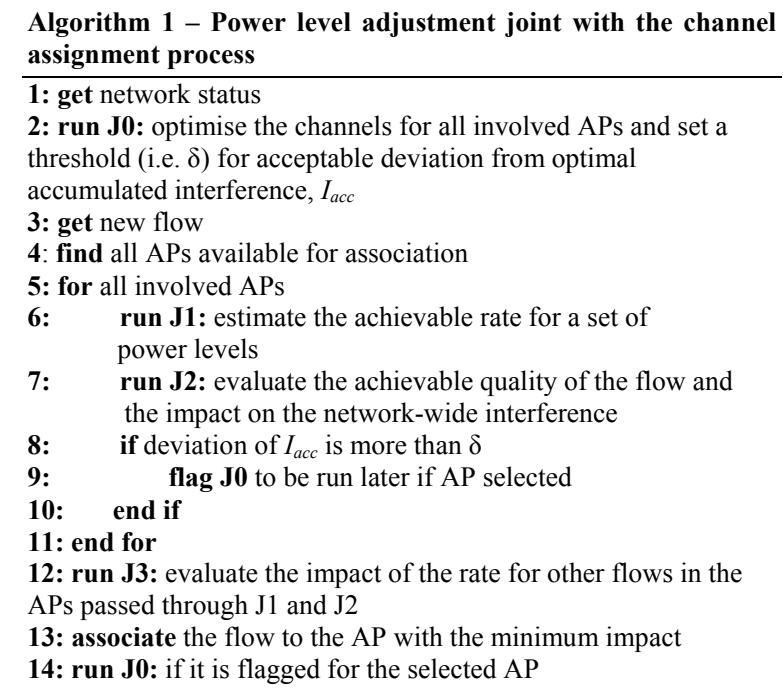

loop' (lines 4-11) is called $N$ times therein lines 6 and 7 are repeated at most $M$ times. Therefore, the time complexity of each run of the setting adjustment for each flow will be $O(M N)$.

\section{EVAluation Scenarios AND Simulation Results}

\section{A. Simulation Setup and Evaluation Strategy}

To evaluate our proposed RRM algorithm, we use MATLAB to simulate the SDWN-based controller in a dense Wi-Fi environment that consists of 25 fixed Wi-Fi APs randomly deployed in an area of $300 \mathrm{~m} \times 300 \mathrm{~m}$ at a minimum distance of $50 \mathrm{~m}$ from each other. The APs transmit power varies from $0 \mathrm{dBm}$ to $40 \mathrm{dBm}$ and is dynamically adjusted during the simulation process. Up to 200 user stations are deployed randomly at a minimum distance of $1 \mathrm{~m}$ from each other and from the APs. The outcome of 10 independent run of each simulation scenario is used to generate the results while the locations of the user stations, APs and their power levels are set different in each run. We adopted a large scale path loss model with the path loss exponent set to 2.5 , a fixed noise level at $99 \mathrm{dBm}$ and the threshold in (4) is set to $-80 \mathrm{dBm}$.

In our evaluation, we first compare the RRM algorithm without transmit power adjustment (i.e. channel assignment only) against the Least Congested Channel algorithm (LCC) [16]. In LCC, each AP acquires a suitable channel based on the neighbouring APs' channels (this can also be implemented based on the contention and retransmission statistics evaluated 


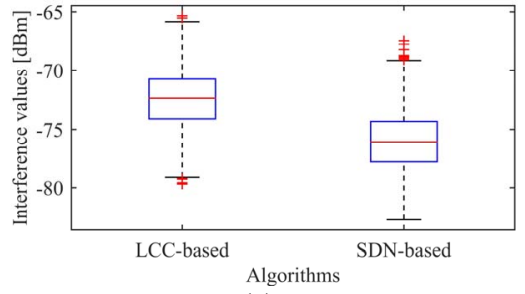

(a)

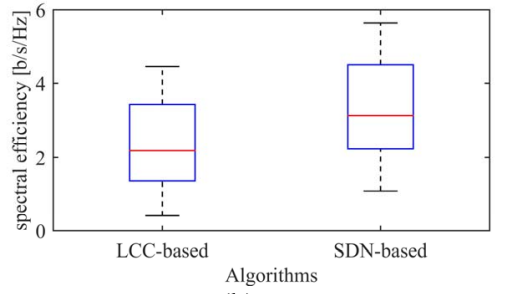

(b)

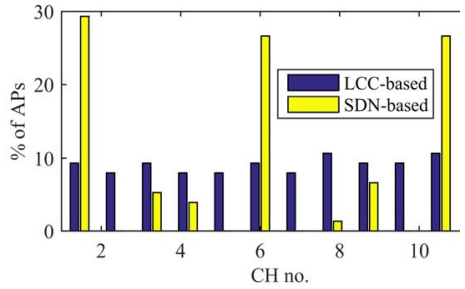

(c)

Fig. 5: the proposed channel assignment process performance

at the MAC layer). LCC represents the basis for the majority of state of the art commercial channel assignment solutions employed in local or central RF channel management. We then compare the results of the channel assignment only algorithm against the combined RRM algorithm, which includes both channel assignment and transmit power adjustment. We also show the possibility of setting a desired threshold to the interference variation as a consequence of power adjustment, beyond which the channel assignment procedure will be triggered, or the intended change will be denied. This is also necessary for reducing traffic interruption due to frequent execution of the RF channel reassignment process.

\section{B. Simulation Results Analysis}

For the first evaluation, we compared the average interference level throughout the network based on LCC and our SDWN-based approach in Fig. 5-(a). The upper and lower edges of the plotted boxes are the 25 th and 75 th percentile of the values and the median values are indicated by the central red lines. The values which we considered as outliers are indicated by red symbols in each case. The results show more than $3 \mathrm{~dB}$ improvement which is reflected in the achievable SINR and subsequently the higher spectral efficiency as shown in Fig. 5(b). An extra $0.8 \mathrm{~b} / \mathrm{s} / \mathrm{Hz}$ improvement with the proposed centralized channel assignment leads to $16 \mathrm{Mb} / \mathrm{s}$ extra capacity achievable at the physical layer and for each employed RF channel throughout the network. This is $25 \%$ of a standard IEEE 802.11g-SISO capacity per channel. Fig. 5-(c) shows the combination of the channels which has been assigned through LCC and our proposed approach. The non-overlapping channels 1,6 and 11 have been used more frequently in our centralised model alongside a limited number of overlapping channels 3,4 , 8 and 9. This combination of overlapping and non-overlapping channels provides the optimal trade-off between co-channel and adjacent-channel interference impacts, given the exemplified network status.

For the second evaluation, we assume two scenarios: Scenario A and Scenario B. In Scenario A, all the flows are transmitted at the default power level (i.e., 20dBm); and Scenario B includes the RRM algorithm with power control where the adaptive power level is used according to each flow's demand (i.e. required bitrate). The power level distribution of the flows in each scenario are presented in Fig. 6-(a), with the transmission power level distribution of Scenario A flows represented in the blue bar, and the power level distribution of Scenario B flows represented in the yellow bars. Fig. 6-(b) shows a comparison of the interference levels measured in the network using the RRM algorithm without and with power control (i.e. channel assignment without and with power control). The results presented in this figure show that the complete RRM algorithm (Scenario B) offers a $15 \mathrm{~dB}$ reduction in the interference over the channel assignment only algorithm (Scenario A).

We also assess the performance of our RRM algorithm with regards to its adaptability to meet the QoS requirements of three classes of flows. Low transmission rate flows: the transmission rate of these flows will vary between $100 \mathrm{Kbps}$ and $1.6 \mathrm{Mbps}$. Medium transmission rate flows: the transmission rate of these flows will vary between $200 \mathrm{Kbps}$ and $3 \mathrm{Mbps}$. High transmission rate flows: the transmission rate of these flows will vary between $500 \mathrm{Kbps}$ and 6Mbps.

A user's demand dependency of the proposed power control mechanism is expected to result in a higher power level when the average demand of the users is high. This has been shown in Fig. 7-(a) where the distribution of adopted power levels are compared for the flows with low and high transmission rates. The higher the average demand, the higher the median of the adopted power levels. However, higher values of transmission power jeopardise network-wide control of the interference level achieved through the optimal channel assignment. Fig. 7-(b) depicts the average interference levels measured in the network after the RRM algorithm is applied for the above three classes of flows. The results presented in this figure show that higher transmission rate flows result in higher interference. This shows that the proposed radio configuration algorithms cannot meet the demands of flows with high rates requirements, without resulting in higher interference levels throughout the network. Subsequently, although the transmit power adjustment will help to increase the transmission rate of a flow, it could result in higher interference levels that degrades the network-wide served quality.

To address this limitation, we propose to apply an upperbound threshold for the maximum acceptable deviation of interference quantity from its optimal value (denoted as $\delta$ in Section IV). The effect of this improvement is shown in Fig. 8 where the optimal value is achieved by applying the channel assignment optimisation algorithm, and deviation from this value is a result of the new flows' corresponding downlink transmission powers. The higher acceptable deviation $(50 \%$ in Fig. 8-(a)) results in a higher network-wide interference compared to the lower acceptable deviation of $5 \%$. Note that in both cases, eventually, the channel assignment process will be triggered to re-adjust the channels. This could be too frequent and disruptive with a very low deviation threshold.

By introducing a threshold on the power level for the sake of interference control, we expect a reduction of the positive correlation between adjusted power levels and the demand of the flows, as shown in Fig. 8-b. This figure shows the correlation 


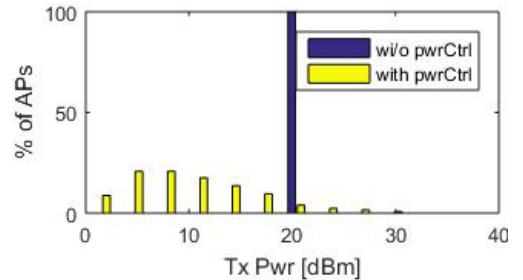

(a)

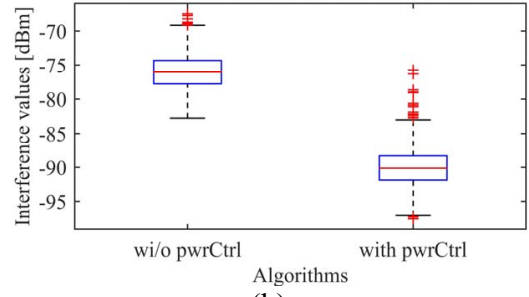

(b)

Fig. 6: power adjustment performance and its interference control impact

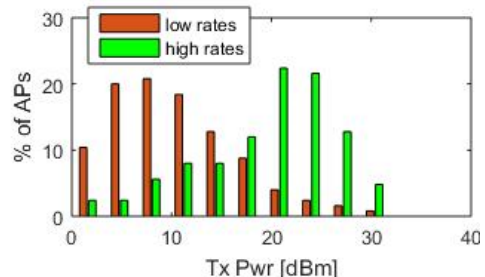

(a)

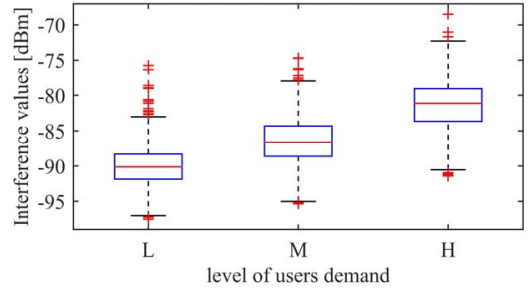

(b)

Fig. 7: interference as a result of the users' demand dependency of power levels

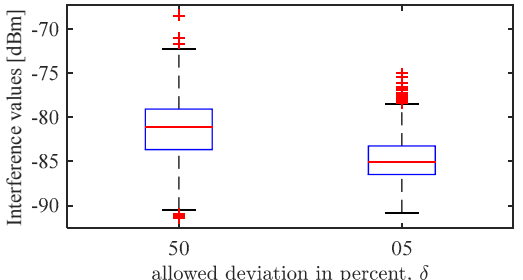

(a)

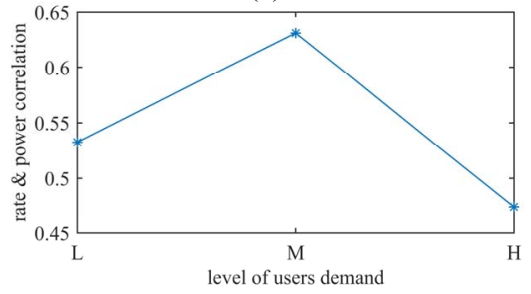

(b)

Fig. 8: correlation between user's demand and power level between the transmit power and the required data rate on one hand and the user demand on the other, for low and medium rate flows. However, this correlation drops when high rate flows are used. This is due to the fact that the RRM tries to control the interference in the network by denying very high transmission power levels.

\section{CONCLUSION}

In this paper we have presented a fine grained radio configuration algorithm that uses transmission power control and channel assignment to address radio interference in dense $\mathrm{Wi}-\mathrm{Fi}$ environments. The proposed algorithm is designed for a SDWN Wi-Fi framework where the controller acts as the central management entity upon which the algorithm is executed. The algorithm relies on network-wide status information as well as flow-based quality demands. The performance of the algorithm has been assessed through simulation and the obtained results show that it provides a lower interference while maintaining the status for a wide range of users' quality demands. This is achieved by taking into account the correlations and mutual relationships between transmission power levels, the required quality of the users and the network-wide interference quantity in the proposed algorithm.

Future work will consider some important characteristics of the use cases such as dynamism in the pattern of the user demand as well as their distribution throughout the network.

\section{ACKNOWLEDGMENT}

This work is funded by the Horizon 2020 Framework Programme of the European Union as part of the Wi-5 project (644262). Further information is available at http://www.wi5.eu/.

\section{REFERENCES}

[1] B. Abeysekera, K. Ishihara, Y. Inoue, M. Mizoguchi, "NetworkControlled Channel Allocation Scheme for IEEE 802.11 Wireless LANs: Experimental and Simulation Study", Vehicular Technology Conference (VTC Spring), Seoul, Korea, 18-21 May 2014.

[2] K. Zhou, X. Jia, L. Xie, Y. Chang, and X. Tang, "Channel Assignment for WLAN by Considering Overlapping Channels in SINR Interference Model", International Conference on Computing, Networking and Communications (ICNC), Maui, Hawaii, USA 30 Jan.- 2 Feb. 2012.
[3] A. Mishra, S. Banerjee, and W. Arbaugh, "Weighted coloring based channel assignment for WLANs." SIGMOBILE Mob. Comput. Commun. Rev. 9(3): 19-31, 2005.

[4] M. Yu, X. Ma, W. Su, and L. Tung, "A new joint strategy of radio channel allocation and power control for wireless mesh networks." Computer Communications 35(2): 196-206, 2012.

[5] Lingzhi Wang, Cunqing Hua, Rong Zheng and Rui Ni, "Online channel selection and user association in high-density WiFi networks," Int. Conf. on Communications (ICC), 8-12 Jun., London, UK, 2015.

[6] K. Ramachandran, R. Kokku, Z. Honghai, and M. Gruteser, "Symphony: Synchronous Two-Phase Rate and Power Control in 802.11 WLANs," Networking, IEEE/ACM Transactions on, vol. 18, pp. 1289-1302, 2010.

[7] Zou, M., et al. "Throughput Improvement of 802.11 Networks Via Randomization of Transmission Power Levels." IEEE Transactions on Vehicular Technology 65(4): 2703-2714, 2016.

[8] V. Navda, R. Kokku, S. Ganguly, S. Das, "Slotted Symmetric Power Control in managed WLANs," Tech. Rep., NEC Lab. America, 2007.

[9] "Software-defined networking: The new norm for networks," Palo Alto, CA, USA, White Paper, Apr. 2012. [Online]. Available: https://www.opennetworking.org/images/stories/downloads/sdnresources/white-papers/wp-sdn-newnorm.pdf.

[10] R. Riggio, T. Rasheed, and F. Granelli, "EmPOWER: A Testbed for Network Function Virtualization Research and Experimentation", IEEE SDN for Future Net. and Services, 11-13 Nov., Trento, Italy, 2013.

[11] J. Schulz-Zander, L. Suresh, N. Sarrar, and A. Feldmann, "Programmatic Orchestration of WiFi Networks," USENIX Symposium on Networked Systems Design and Implementation (NSDI), Philadelphia, PA, USA, 1920 Jun. 2014

[12] F. Bouhafs (Editor), "Wi-5 initial architecture", Deliverable D2.4 of Wi5 project, Dec. 2015, available at http://www.wi5.eu/.

[13] J. Saldana (Editor), "Specification of Smart AP solutions version 1", Deliverable D3.2 of Wi-5 project, Dec. 2015, available at http://www.wi5.eu/.

[14] A. Raschellà, F. Bouhafs, M. Seyedebrahimi, M. Mackay, Q. Shi, "A Centralized Framework for Smart Access Point Selection based on the Fittingness Factor", International Conference on Telecommunications (ICT), Thessaloniki, Greece, 16-18 May, 2016.

[15] M. Seyedebrahimi, F. Bouhafs, A. Raschellà, M. Mackay, Q. Shi, "SDNBased Channel Assignment Algorithm for Interference Management in Dense Wi-Fi Networks", European Conf. on Networks and Communications (EuCNC), Athens, Greece, 27-30 June, 2016.

[16] M. Achanta, "Method and Apparatus for Least Congested Channel Scan for Wireless Access Points," US Patent No. 20060072602, Apr. 2006. 